\title{
Perceived Social Support/Conflict
}

National Cancer Institute

\section{Source}

National Cancer Institute. Perceived Social Support/Conflict. NCI Thesaurus. Code C93218.

An individuals' perception of the amount of positive support (e.g., love, care, concern) and negative support (e.g., demand, critique) that they experience. 